\title{
Accumulation of natural killer cells in ischemic brain tissues and the chemotactic effect of IP-10
}

\author{
Yao Zhang ${ }^{1 \dagger}$, Zhongming Gao ${ }^{1,2 \dagger}$, Dandan Wang ${ }^{1}$, Tongshuai Zhang ${ }^{1}$, Bo Sun ${ }^{1}$, Lili Mu', Jinghua Wang ${ }^{1}$, \\ Yumei Liu', Qingfei Kong ${ }^{1}$, Xijun Liu', Yue Zhang ${ }^{1,5}$, Haoqiang Zhang ${ }^{1}$, Jiqing He ${ }^{1,2}$, Hulun Li ${ }^{1,3^{*}}$ \\ and Guangyou Wang ${ }^{1,3,4^{*}}$
}

\begin{abstract}
Background: Stroke is accompanied by a distinguished inflammatory reaction that is initiated by the infiltration of immunocytes, expression of cytokines, and other inflammatory mediators. As natural killer cells (NK cells) are a type of cytotoxic lymphocyte critical to the innate immune system, we investigated the mechanism of NK cells-induced brain injuries after cerebral ischemia and the chemotactic effect of IP-10 simultaneously.

Methods: NK cells infiltration, interferon-gamma (IFN- - ) and IP-10 expression were detected by immunohistochemistry, immunofluorescence, PCR and flow cytometry in human and C57/BL6 wild type mouse ischemic brain tissues. The ischemia area was detected via 2,3,5-triphenyltetrazolium chloride staining. CXCR3 mean fluorescence intensity of isolated NK cells was measured by flow cytometry. The neuronal injury made by NK cells was examined via apoptosis experiment. The chemotactic of IP-10 was detected by migration and permeability assays.

Results: In human ischemic brain tissue, infiltrations of NK cells were observed and reached a peak at 2 to 5 days. In a permanent middle cerebral artery occlusion (PMCAO) model, infiltration of NK cells into the ischemic infarct region reached their highest levels 12 hours after ischemia. IFN-Y-positive NK cells and levels of the chemokine IP-10 were also detected within the ischemic region, from 6 hours up to 4 days after pMCAO was performed, and IFN- $\gamma$ levels decreased after NK cells depletion in vivo. Co-culture experiments of neural cells with NK cells also showed that neural necrosis was induced via IFN- - . In parallel experiments with IP-10, the presence of CXCR3 indicates that NK cells were affected by IP-10 via CXCR3, and the effect was dose-dependent. After IP-10 depletion in vivo, NK cells decreased. In migration assays and permeability experiments, disintegration of the blood-brain barrier (BBB) was observed following the addition of NK cells. Moreover, in the presence of IP-10 this injury was aggravated.
\end{abstract}

Conclusions: All findings support the hypothesis that NK cells participate in cerebral ischemia and promote neural cells necrosis via IFN- - . Moreover, IP-10 intensifies injury to the BBB by NK cells via CXCR3.

Keywords: NK cells, Cerebral ischemia, IP-10, CXCR3

\section{Background}

The immune system and the central nervous system (CNS) can affect one another both locally and systemically. For example, during a stroke, the immune response of the CNS induces activation of resident glia cells and macrophage [1-4],

\footnotetext{
*Correspondence: lihulun@aliyun.com; guangyouwang@163.com

${ }^{\dagger}$ Equal contributors

'Department of Neurobiology, Harbin Medical University Provincial Key Lab of Neurobiology, Harbin Medical University, Xuefu Road, 150081, Heilongjiang,

People's Republic of China

${ }^{3}$ The Key Laboratory of Myocardial Ischemia, Harbin Medical University, Ministry of Education, Xuefu Road, 150001, Heilongjiang, Province People's Republic of China Full list of author information is available at the end of the article
}

as well as the infiltration of circulating immune cells, respectively. A stroke is hypothesized to be a multiphasic process [5] and, in most cases, the cause of death results from inflammation without exogenous infection. Thus, a secondary component of ischemic brain injury may involve the effects of a significant inflammatory response.

In the first hours following stroke, rapid activation of resident microglia and production of pro-inflammatory cytokines occurs [6]. In addition, neutrophils and monocytes and/or macrophage infiltrate and accumulate in microvessels and in the ischemic cerebral parenchyma [7]. Accumulating evidence further suggests that the resulting 
inflammation response is responsible for inducing cerebral injury that is secondary to the hypoxia and ischemia that predominantly characterize a stroke event [8]. However, it is important to note that the action of the immune system is not uniform, and activation of immune cells may play different roles at different times.

Natural killer (NK) cells are a type of cytotoxic lymphocytes that play an important role in the innate immune system. For example, NK cells mediate a response to virally infected cells within three days of infection, and respond to tumor formation. The term 'natural killers', was coined based on the initial notion that these lymphocytes do not need to be activated to kill cells [9]. NK cells are key components of the innate immune system since they are able to rapidly produce an abundance of cytokines, mainly IFN- $\gamma$, and are able to lyse target cells without prior sensitization $[10,11]$. The role of NK cells in the development of adaptive immune responses has also recently been shown [12].

Numerous observations have suggested that NK cells play a role in the adaptive immune response associated with autoimmune diseases. For example, the expansion of blood NK cells has been found to correlate with the suppression of disease activity, and NK cells isolated from patients during treatment were found to be highly activated [13-15].

While stroke is a disease that directly affects the neural system, it is also a type of autoimmune disease involving non-infection induced inflammation. Peterfalvi et al. [16] proved that NK cells and its fundamental cytokines decrease after stoke in human peripheral circulation. As cerebral ischemia is a kind of focal disease, focusing on changes in central system becomes necessary. Therefore, the objective of this study was to investigate the possible role of NK cells in stroke, including the timing of their infiltration into the brain, whether they mediate beneficial or harmful effects, what kind of role they may have in cerebral ischemia, and what kind of changes NK cells undergo during this process. Accordingly, functional alterations in cytokine and chemokine production by NK cells in both human and mouse models during the acute phase of ischemic stroke were examined.

\section{Methods}

\section{Animal experiments}

Adult male C57/BL6 mice (weighing 20 to $25 \mathrm{~g}$ ) were obtained from Peking Vital River Laboratory Animal Ltd (Beijing, People's Republic of China). All mice were bred and maintained in accordance with the guidelines of the Care and Use of Laboratory Animals published by the China National Institute of Health. Furthermore, all experiments were conducted in accordance with institutional guidelines and were approved by Harbin Medical University ethics committee. A permanent middle cerebral artery occlusion (pMCAO) mouse model was established using the intraluminal filament method (6-0 nylon) as previously described [17]. In the sham group arteries were blunt dissected, yet not ligated. Following surgery, each mouse was assessed on a scale from 0 to 5 upon awakening, and only mice receiving a score of $>1$ were included in this study [18].

\section{Immunohistochemistry and immunofluorescence staining for NKp46, IP-10, CXCR3 and IFN- $\gamma$}

Human samples were provided by the Department of Neurosurgery, The First Clinical College of Harbin Medical University and were used for our research which was approved by the Harbin Medical University ethics committee. Patient deaths were identified as due to cerebral ischemic infarction and were diagnosed using computerized tomography. Tissue samples were taken from an area adjacent to the infarct site, and also from a similar area on the normal contralateral hemisphere, from each human brain sample. Tissues were embedded in paraffin and sectioned $(10 \mu \mathrm{m})$. Frozen CNS material from mouse models were obtained after autopsy. Briefly, after being fixed in cold acetone for 20 minutes, tissues were blocked in $5 \%$ horse serum for 1 hour at room temprature, and were then permeabilized with $0.1 \%$ Triton X-100 (Sigma, St. Louis, USA) for 30 minutes. Primary antibodies used included: goat anti-mouse/human NKp46 (1/150, Santa Cruz, Dallas, USA), goat anti-mouse/human IP-10 (1/150, Santa Cruz, Dallas, USA), rabbit anti-mouse CXCR3 (1/ 150, Santa Cruz, Dallas, USA), and rat anti-mouse IFN- $\gamma$ (1/150, Biolegend, San Diego, USA). Tissues were incubated with antibodies overnight at $4^{\circ} \mathrm{C}$. After several washes with phosphate buffing saline (PBS), the appropriate secondary antibodies were applied (1:200, Zhongshan Goldenbridge Biotechnology Co. Ltd., Beijing, People's Republic of China). Nuclei were co-stained with 4,6-diamidino-2-phenylindole (DAPI). Control stainings included the omission of primary antibody. The human tissue section areas were measured by image analysis software (Image Pro Plus 5.0, Media Cybernetics, Warrendale, USA). The numbers of stained cells per $\mathrm{mm}^{2}$ of tissue area were calculated.

\section{2,3,5-triphenyltetrazolium chloride staining}

Mice were euthanized 12 hours after pMCAO under deep anesthesia with sodium pentobarbital $(60 \mathrm{mg} / \mathrm{kg}$ body weight). Brains were rapidly removed after intracardial infusion of PBS. The brains were then cut on the coronal suture into five slices, using a brain slicer. Brain slices were immediately incubated in 2,3,5-triphenyltetrazolium chloride (TTC) solution (2\% solution in PBS) for 30 minutes at $37^{\circ} \mathrm{C}$. Areas not stained red with TTC were considered to be damaged. 


\section{Primary cultures of neural cells}

Neural cell cultures were prepared from newborn C57/ BL6 mice. Soon after discarding the meninges, the mesencephalon, cerebellum, and brain stem, cortexes were blunt isolated from the cerebrum and then gently pipetted to achieve a single cell suspension in 10\% DMEM (supplemented with $10 \%$ FCS, $100 \mathrm{U} / \mathrm{ml}$ penicillin, $0.1 \mathrm{mg} / \mathrm{ml}$ streptomycin). Cell suspensions were passed through a $150 \mu \mathrm{m}$ nylon filter, then were seeded in 24-well plates $\left(5 \times 10^{4}\right.$ cells/well $)$ or $25 \mathrm{~mm}^{2}$ flasks $\left(10^{5} / \mathrm{ml}\right)$ coated with $\mathrm{D}$-polylysine (PDL), and were cultured for at least 7 days.

\section{Flow cytometry}

Mice were euthanized and perfused with PBS. Hemispheres were divided to isolate non-ischemic (ipsilesional) and ischemic (contralesional) tissue samples. Three to four hemispheres were pooled for each treatment group, and the resulting homogenates were pressed through $150 \mu \mathrm{m}$ and $75 \mu \mathrm{m}$ filters. Next, cells were separated from myelin and debris using 70\% and 30\% Percoll (GE Healthcare, Pittsburgh, USA) gradients, respectively, followed by centrifugation. Samples were then incubated with anti-mouse NKp46 antibody (Santa Cruz, Dallas, USA) for 30 minutes at RT in a fluorescence-activated cell sorter (FACS) buffer ( $0.1 \%$ bovine serum albumin (BSA), $0.01 \%$ sodium azide in PBS). After the cells were washed twice with wash buffer (0.1\% BSA/PBS), FITC-conjugated anti-goat secondary antibodies (Biolegend, San Diego, USA) were applied for 30 minutes at RT. Finally, the cells were fixed with $2 \%$ paraformaldehyde at $4^{\circ} \mathrm{C}$, and were then analyzed by blinded evaluators using a FacsCalibur (BD Biosciences, Franklin Lakes, USA) and FlowJo software (ThreeStar Inc., Ashland, USA) Three independent experiments were performed for all time points, and 3 to 4 mice were used per experiment.

Neural cells for apoptosis detection were prepared from newborn C57/BL6 mice as previously described, and were cultured in 10\% DMEM until they covered the bottom of a $25 \mathrm{~mm}^{2}$ flask. Cells were then treated with NK cells $\left(10^{5}\right)$ harvested from C57/BL6 12 weeks mice' spleens with a MagCellect Mouse NK Cell Isolation Kit (R\&D, Minneapolis, USA) and murine IFN- $\gamma$ neutralizing antibody (10 ng/ml, R\&D, Minneapolis, USA) in $10 \%$ DMEM, then stained with a PI/Annexin kit (BD Biosciences, Franklin Lakes, USA). Stained cells were detected by evaluators who have no relationship with this experiment using a FacsCalibur (BD Biosciences, Franklin Lakes, USA) and FlowJo software. Three independent experiments were performed.

NK cells harvested from 12-week old C57/BL6 mice spleens with a MagCellect Mouse NK Cell Isolation Kit (R\&D, Minneapolis, USA) were treated with murine IP-10 (10 ng/ml, $50 \mathrm{ng} / \mathrm{ml}, 100 \mathrm{ng} / \mathrm{ml})$ accompanied by 6 hour oxygen glucose deprivation (OGD) in
Hank's Balanced Salt Solution (HBSS) (Ca-Mg-free, glucose $1 \mathrm{~g} / \mathrm{L}$ ). Mean fluorescence of CXCR3 (PE) which NK cells presented were blinded evaluators using a FacsCalibur (BD Biosciences, Franklin Lakes, USA) and FlowJo software. Three independent experiments were performed. Non-OGD groups were cultured in a cell incubator $(5 \%$ $\mathrm{CO}_{2}$ ) in HBSS (Ca-Mg-free, $1 \mathrm{~g} / \mathrm{L}$ glucose). Three independent experiments were performed.

\section{Reverse transcription PCR}

Total RNA from the whole cortex, which had been sorted in pMCAO mice, was obtained at different time points using a Trizol extraction method recommended by Invitrogen. Reverse transcription was performed using an RT-PCR kit from TaKaRa (Kusatsu, Japan). PCR amplification of IP10 ,IFN- $\gamma$, and glyceraldehyde-3-phosphate dehydrogenase (GAPDH) (endogenous control) were achieved using Taq polymerase and the following primers: IP- 10 sense $5^{\prime}-\mathrm{GCC}$ GTC ATT TTC TGC CTC AT-3' and anti-sense 5'-GCT TCC CTA TGG CCC TCA TT-3', IFN- $\gamma$ sense 5'-AGC GGC TGA CTG AAC TCA GAT TGT-3' and anti-sense 5'-GTC ACA GTT TTC AGC TGT ATA GGG-3', and GAPDH sense 5'-AAT GCA TCC TGC ACC AA-3' and anti-sense $5^{\prime}$-TCC ACC ACC CTG TTG CTG TA-3'. A total of 40, 40 and 32 cycles were performed for IP-10, IFN- $\gamma$ and GAPDH, respectively. Three independent experiments were performed.

\section{Migration assays}

Neural cells $\left(5 \times 10^{4}\right.$ cells) prepared from newborn C57/ BL6 mice as previously described were seeded in the bottom of 24-well transwell plates. After culturing the cells for at least 7 days, mouse brain endothelial cells (bEnd3, ADCC) $\left(1 \times 10^{4}\right)$ were plated on top of $2 \%$ gelatincoated $3 \mu \mathrm{m}$ pore size upper transwell chambers with $10 \%$ DMEM media. After the ECs formed a confluent monolayer, $20 \mathrm{ng} / \mathrm{ml}$ murine IP-10 neutralization antibody (R\&D, Minneapolis, USA) was added to the lower chambers when appropriate. Simultaneously, a suspension of NK cells $\left(1 \times 10^{6} / \mathrm{ml} ; 100 \mu \mathrm{l}\right)$ prepared from 12 -week old C57/BL6 mice spleens with a MagCellect Mouse NK Cell Isolation Kit (R\&D, Minneapolis, USA) were added to the upper chamber. The ability of NK cells to cross the monolayer was evaluated by counting the absolute number of cells that migrated to the lower chamber after OGD conditions were applied for 6 hours in HBSS (Ca-Mg-free, $1 \mathrm{~g} / \mathrm{L}$ glucose). Furthermore, the cells in the upper chamber were indirectly labeled with NKp46-FITC, cell counts were performed using FACS, and three independent experiments were performed. Non-OGD groups were cultured in a cell incubator $\left(5 \% \mathrm{CO}_{2}\right)$ in $\mathrm{HBSS}$ ( $\mathrm{Ca}$ Mg-free, $1 \mathrm{~g} / \mathrm{L}$ glucose). 


\section{Permeability assays}

Permeability assays were conducted as previously described [19]. Soon after 6 hours OGD in HBSS (Ca-Mg-free, $1 \mathrm{~g} / \mathrm{L}$ glucose), $50 \mu \mathrm{g} / \mathrm{ml}$ fluorescein isothiocyanate-labeled BSA (FITC-BSA, Sigma, St. Louis, USA) was added to the upper chamber of transwells. Samples (both $50 \mu \mathrm{l}$ ) were then obtained from the upper and lower chambers of each well and the fluorescence intensity of these samples was measured using a FL600 microplate fluorescent reader (Biotek, Vermont, USA). The diffusion rate, representing the permeability of blood brain barrier (BBB) endothelial cells (ECs), was expressed as a percentage and was calculated as follows: (BSA lower chamber) $\times 100$ / (BSA upper chamber). Non-OGD groups were cultured in a cell incubator $\left(5 \% \mathrm{CO}_{2}\right)$ in HBSS (Ca-Mg-free, $1 \mathrm{~g} / \mathrm{L}$ glucose).Three independent experiments were performed.

\section{In vivo NK depletion assay}

Depletion of NK cells in vivo in mice was induced by intraperitoneal injection (i.p.) of a depleting anti-NK1.1 mAb (clone PK136, Biolegend, San Diego, USA). The depleting antibody was given at $0.5 \mathrm{mg}$ i.p. 1 day before the pMCAO mouse model (12 hours) was established as previously described. The lymphocytes infiltration assay was then performed via FACS, and IFN- $\gamma$ expression level was detected by RT-PCR as previously described.

\section{In vivo IP-10 depletion assay}

To block IP-10 in vivo, mice were injected intravenously with $3 \mathrm{mg} / 100 \mathrm{~g}$ body weight anti-mouse IP-10 (R\&D Systems) for two days to neutralize the IP-10 secretion before the pMCAO mouse model was established as previously described, and the lymphocytes infiltration assay was then performed via FACS.

\section{Statistical analysis}

Results are expressed as the mean \pm standard deviation (SD). An analysis of variance (ANOVA) test was used to compare multiple quantitative variables. Statistical analysis was performed using one-way ANOVA analysis of variance followed by Student's t-test. Statistical significance was considered to be $P<0.05$.

\section{Results}

\section{NK cells participate in cerebral ischemia}

Paraffin-embedded human brain tissues affected by cerebral ischemic infarction, as well as contralateral tissues, were divided into three groups according to the period of time that elapsed between the onset of stroke and death. These groups included: $<2$ days, 2 to 5 days, and $>5$ days, with 5,11 , and 4 sets of patient tissues included in each group, respectively (Table 1, 1-20). Immunohistochemistry staining of NK cells was performed for each tissue set (Figure 1A,B), and the number of NK cells in the ischemic hemispheres was found to be significantly greater than those in the non-ischemic hemispheres $\left(P_{<2}\right.$ days $<$ $0.05, P_{2}$ to 5 days $\left.<0.01, P_{>5 \text { days }}<0.05\right)$. Furthermore, the highest numbers of NK cells were observed in the 2 to 5 days tissues (Figure 1C).

Next, the infiltration of NK cells was examined in a C57/BL6 pMCAO model. NK cells were detected in ischemic brain tissues, and these cells were consistently observed in proximity of the ischemic penumbra (Figure 1D,E). To monitor NK cells during ischemia, infiltrating cells were isolated from the cerebrum at various timepoints and were analyzed by flow cytometry. Based on the FACS data obtained, the infiltration of NK cells peaked 12 hours after ischemia, and the number of NK cells in the ischemic hemisphere was significantly greater than that of the non-ischemic hemisphere $12,24,48$, and 96 hours after ischemia $\left(P_{12}\right.$ hours $<0.01$, $P_{24} \quad$ hours $<0.001, \quad P_{48} \quad$ hours $<0.05, \quad P_{96} \quad$ hours $\left.<0.05\right)$ (Figure 1F). Moreover, the percentage of NK cells among the infiltrating cells recovered was highest 12 hours after ischemia, and decreased at the subsequent timepoints (Figure 1G). After NK cells depletion, NK cells infiltration notably decrease in ischemic mouse brain at 12 hours (Figure $1 \mathrm{H}, P<0.001$ ), and the ischemia area diminished with TTC staining (Figure 1I).

\section{NK cells negatively affect neural cells via IFN- $\gamma$}

IFN- $\gamma$ is one of the main cytokines secreted by NK cells. Using immunofluorescence assays and FACS, IFN- $\gamma-$ positive NK cells present in ischemic tissues were analyzed 12, 24, 48, and 96 hours after ischemia (Figure 2A,B). The highest levels of IFN- $\gamma$-positive NK cells were detected 12 and 72 hours after ischemia $\left(P_{12}\right.$ hours $<0.01, P_{72}$ hours $<$ 0.01) (Figure 2C).

Staining for apoptosis and necrosis, we found levels of necrosis of neural cells significantly increased after adding NK cells, compared with the control group (only neural cells), meanwhile levels of apoptosis were quite low $(P<0.001)$. However, after treatment with IFN- $\gamma$ blockade, levels of apoptosis significantly increased and levels of necrosis became lower in the NK + IFN- $\gamma$ blockade group, compared to the NK co-culture group $\left(P_{N}<0.01, P_{A}<\right.$ 0.001 ), while levels of necrosis and apoptosis did not differ from the control group (Figure 2D,E). After NK cells depletion, the level of IFN- $\gamma$ mRNA expression decreased at 12 hours (Figure 2F).

\section{Chemotactic effect of IP-10 on NK cells via CXCR3 during cerebral ischemia}

A second set of human brain tissue samples were obtained, and these derived from patients that died $<7$ days, between 7 and 14 days, or $>14$ days after experiencing an ischemic event. There were four, five, and four tissue sets for each group, respectively (Table 1, 21-33). 
Table 1 Clinical data from stroke patients

\begin{tabular}{|c|c|c|c|c|c|c|}
\hline Number & $\begin{array}{c}\text { Serial } \\
\text { number }\end{array}$ & Gender & Age & Diagnosis & $\begin{array}{l}\text { First or recurrent cerebrovascular } \\
\text { accident }\end{array}$ & Medications \\
\hline 1 & A223 & Male & 60 & Ischemic stroke & First & $\begin{array}{c}\text { Mannitol, Lasix, buffered glycerol saline, Coraminum, lobeline, glucose, insulin, mefoxinvial, } \\
\text { albumin, urokinase }\end{array}$ \\
\hline 2 & A593 & Female & 65 & Ischemic stroke & Recurrent & $\begin{array}{c}\text { Mannitol, Xuesaitong injection, CDPC, urokinase, Dextran, insulin, cephalosporin, Coraminum, } \\
\text { lobeline }\end{array}$ \\
\hline 3 & A437 & Female & 48 & Ischemic stroke & First & Mannitol, Lasix, buffered glycerol saline, Coraminum, lobeline, glucose, insulin, mefoxin vial \\
\hline 4 & A225 & Female & 63 & Ischemic stroke & Recurrent & mannitol, Lasix, Dexamethasone, Saline, Sodium Valproate \\
\hline 5 & A351 & Female & 44 & Ischemic stroke & First & Saline, ahylysantinfarctase \\
\hline 6 & A368 & Male & 65 & Ischemic stroke & First & mannitol, Lasix, Coraminum, lobeline \\
\hline 7 & A375 & Male & 60 & Hemorrhagic stroke & First & mannitol , PAMBA, Lasix, cedilanid \\
\hline 8 & A388 & Female & 56 & Ischemic stroke & First & $\begin{array}{l}\text { Mannitol, Lasix, buffered glycerol saline, urokinase, glucose, cephalosporin, } \\
\text { Dextran, CompoundDanshen, Nao Mai Tong recipe, Coraminum, lobeline }\end{array}$ \\
\hline 9 & A389 & Female & 68 & Ischemic stroke & First & Mannitol, Lasix, buffered glycerol saline, Coraminum, lobeline \\
\hline 10 & A405 & Female & 56 & Ischemic stroke & First & $\begin{array}{l}\text { Mannitol, Lasix, buffered glycerol saline, Sodiumcytidine diphosphate } \\
\text { choline injection, cephalosporin, Coraminum, lobeline, insulin }\end{array}$ \\
\hline 11 & A422 & Male & 43 & Ischemic stroke & First & $\begin{array}{l}\text { cidilaniol, glucose, injcinetodini, coramini, lobeline, Saline, dopamim, } \\
\text { aramim, ritalim, atropiai, epinephrine, sodium bicarbonate, noradrenaline }\end{array}$ \\
\hline 12 & A432 & Male & 64 & Ischemic stroke & Recurrent & $\begin{array}{l}\text { Saline, ahylysantinfarctase, mannitol, Lasix, Coraminum, glucose, injcinetodini, coramini, lobeline, } \\
\text { Saline, dopamim, aramim, ritalim, atropiai, epinephrine, sodium bicarbonate, noradrenaline }\end{array}$ \\
\hline 13 & A597 & Male & 64 & Ischemic stroke & Recurrent & $\begin{array}{c}\text { Mannitol , Xuesaitong injection, CDPC, urokinase, Dextran, insulin, cephalosporin, Coraminum, } \\
\text { lobeline }\end{array}$ \\
\hline 14 & A598 & Male & 48 & Ischemic stroke & First & Mannitol, Lasix, buffered glycerol saline, Coraminum, lobeline, glucose, insulin, mefoxin vial \\
\hline 15 & A599 & Male & 67 & Ischemic stroke & Recurrent & Mannitol, Lasix, buffered glycerol saline, Coraminum, lobeline, glucose, mefoxinvial, albumin \\
\hline 16 & A600 & Female & 61 & Ischemic stroke & First & $\begin{array}{c}\text { Mannitol, Lasix, buffered glycerol saline, Coraminum, lobeline, glucose, insulin, mefoxinvial, } \\
\text { albumin, urokinase }\end{array}$ \\
\hline 17 & A601 & Male & 67 & Ischemic stroke & First & $\begin{array}{c}\text { Mannitol, Lasix, buffered glycerol saline, Coraminum, lobeline, glucose, mefoxinvial, urokinase, } \\
\text { Losec, Cimetidine }\end{array}$ \\
\hline 18 & A602 & Male & 67 & Ischemic stroke & First & Mannitol, Lasix, Nimotop, Aspirin, \\
\hline 19 & A610 & Male & 63 & Ischemic stroke & Recurrent & Insulin, glucose, \\
\hline 20 & A625 & Female & 63 & Ischemic stroke & First & $\begin{array}{c}\text { Mannitol, Lasix, buffered glycerol saline, Coraminum, lobeline, glucose, insulin, mefoxinvial, } \\
\text { albumin, urokinase }\end{array}$ \\
\hline 21 & A634 & Female & 70 & Ischemic stroke & First & Mannitol, Lasix, urokinase, Nitroprusside, insulin, insulin, dopamim \\
\hline 22 & A647 & Male & 60 & Ischemic stroke & First & Nimotop, Losec, Coraminum, lobeline, Citicoline, mannitol \\
\hline 23 & A648 & Female & 47 & Ischemic stroke & First & Lasix, Luminal, urokinase, insulin, glucose, $\mathrm{KCl}, \mathrm{NaCl}$ \\
\hline 24 & A655 & Male & 66 & Ischemic stroke & Recurrent & $\begin{array}{l}\text { Saline, ahylysantinfarctase, mannitol, Lasix, Coraminum, glucose, injcinetodini, coramini, } \\
\text { lobeline, Saline, dopamim, ritalim, atropiai, sodium bicarbonate, noradrenaline }\end{array}$ \\
\hline 25 & 6571 & Female & 53 & Ischemic stroke & First & \\
\hline
\end{tabular}


Table 1 Clinical data from stroke patients (Continued)

\begin{tabular}{|c|c|c|c|c|c|c|}
\hline & & & & & & $\begin{array}{c}\text { Mannitol, Lasix, buffered glycerol saline, urokinase, glucose, cephalosporin, Dextran, } \\
\text { Coraminum, lobeline }\end{array}$ \\
\hline 26 & 6572 & Female & 56 & Ischemic stroke & First & $\begin{array}{l}\text { Mannitol, Lasix, buffered glycerol saline, urokinase, glucose, cephalosporin, } \\
\text { Dextran, CompoundDanshen, Coraminum, lobeline }\end{array}$ \\
\hline 27 & 6573 & Female & 73 & Ischemic stroke & First & Mannitol, Lasix, urokinase, Nitroprusside, dopamim \\
\hline 28 & 6574 & Male & 62 & Ischemic stroke & Recurrent & Mannitol, Lasix, urokinase, insulin, glucose, \\
\hline 29 & 6575 & Male & 59 & Hemorrhagic stroke & First & Mannitol, PAMBA, Lasix, cedilanid \\
\hline 30 & 6576 & Male & 66 & Ischemic stroke & Recurrent & Mannitol, Xuesaitong injection, urokinase, Dextran, insulin, cephalosporin, Coraminum, lobeline \\
\hline 31 & 6577 & Female & 49 & Ischemic stroke & First & Mannitol, Lasix, buffered glycerol saline, Coraminum, lobeline, glucose, insulin \\
\hline 32 & 6578 & Male & 64 & Ischemic stroke & Recurrent & Mannitol, Lasix, buffered glycerol saline, insulin, glucose \\
\hline 33 & 6579 & Female & 45 & Ischemic stroke & First & Mannitol, Lasix, buffered glycerol saline, Coraminum, lobeline, glucose, insulin, mefoxin vial \\
\hline
\end{tabular}



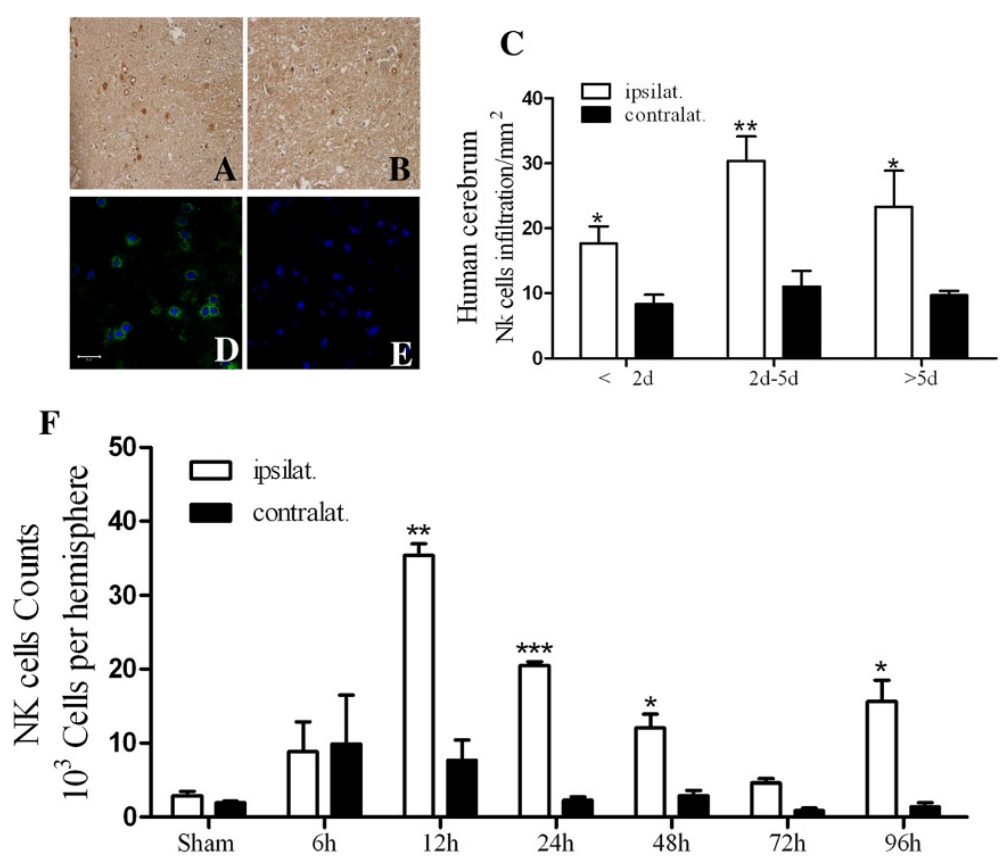

G

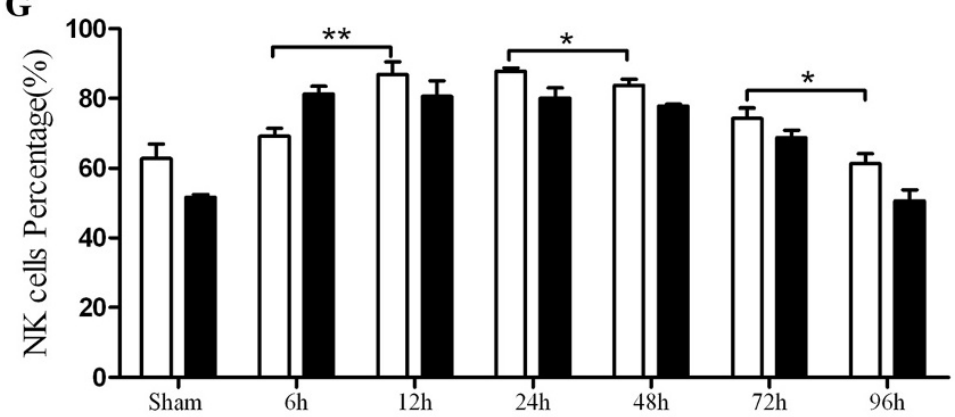

H

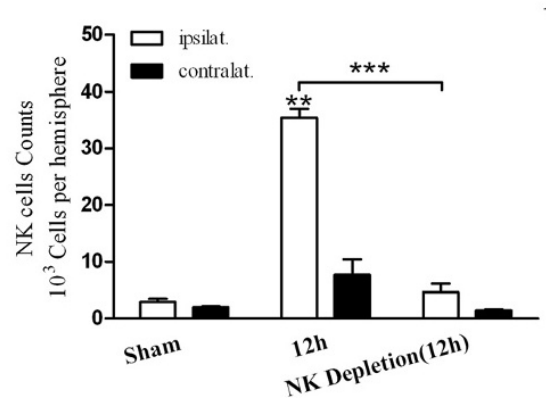

I

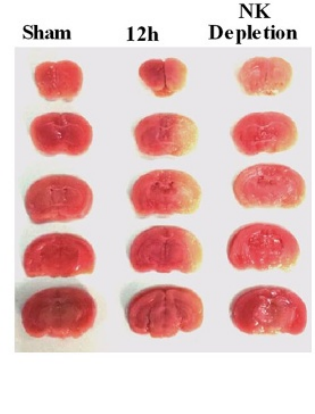

Figure 1 NK cells participate in cerebral ischemia. (A,B): NK cells stained brown in ischemic tissues (magnification, 400x). Differences between the ischemic (A) and non-ischemic (B) hemispheres were significant for the 2 to 5 day group, and were fewer in the non-ischemic hemispheres; (C): Infiltration of NK cells into human ischemic tissue. The highest number of NK cells were detected in the 2 to 5 day group; (D,E): NK cells in mouse ischemic tissue penumbra. NK positive cells (FITC) were found in ischemic tissues (D) $(b a r=30 \mu m)$; (F): Infiltration of NK cells detected in mouse ischemic tissue by FACS; (G): Of the infiltrating lymphocytes detected, the percentage of NK cells is reported; (H): After NK cells depletion in vivo, infiltration of NK cells detected in mouse ischemic tissue by FACS; (I): Representative TTC-stained sections of NK cells depletion mouse at 12 hours following pMCAO. ( ${ }^{*} P<0.05,{ }^{* *} P<0.01,{ }^{* * *} P<0.001$, compared with the corresponding values for the contralateral hemispheres). PMCAO, permanent middle cerebral artery occlusion.

Immunohistochemistry assays were performed, including the staining of IP-10 (Figure 3A,B). A significantly higher number of IP-10-positive cells were detected in the ische- mic hemispheres compared to the contralateral hemispheres among these three groups. In addition, IP-10-positive cells in the ischemic hemisphere tissues of the $<7$ day group 


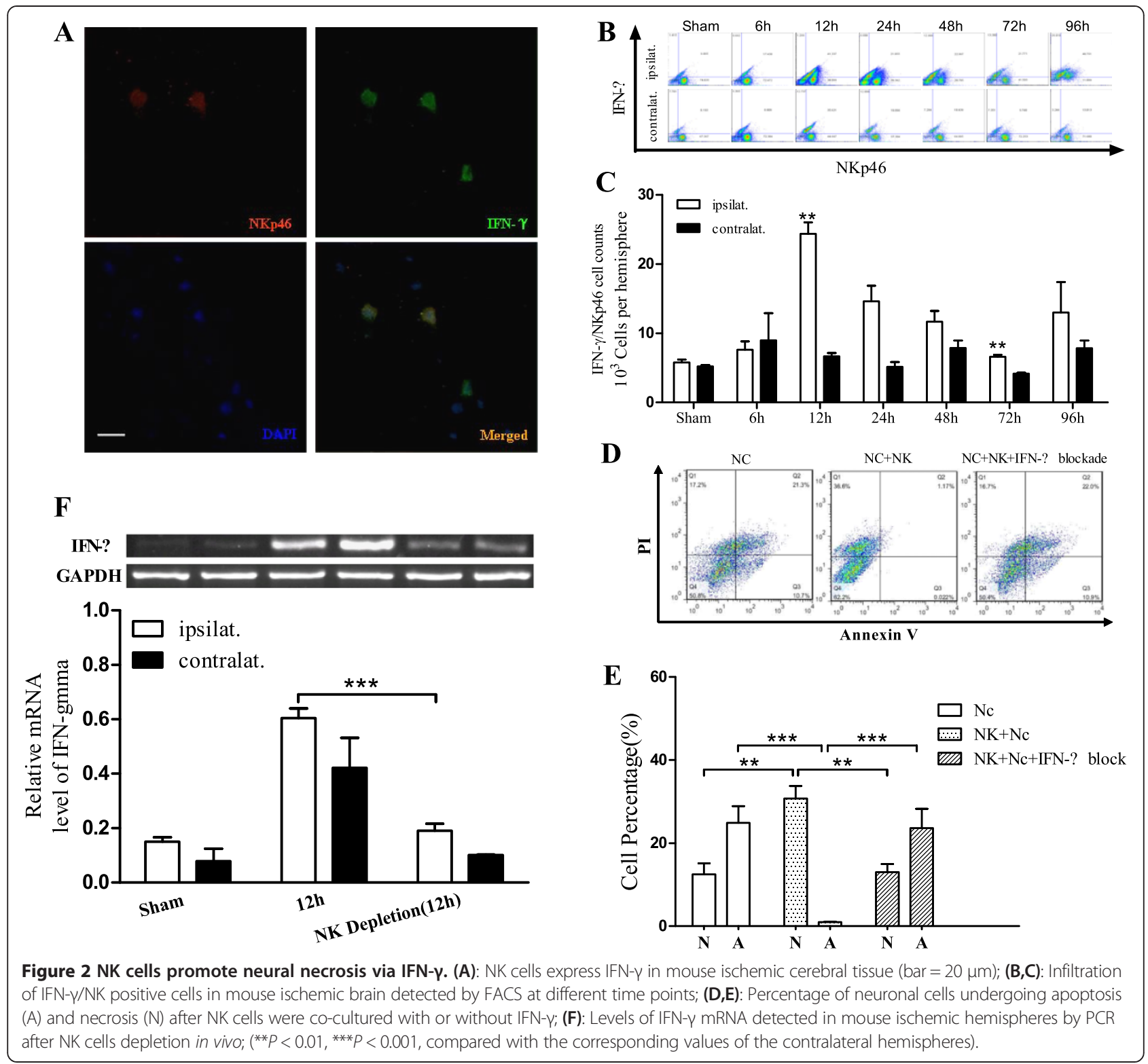

were very high, and the IP-10-positive cells in the ischemic hemisphere tissues of the 7 to 14 days group were present at lower levels compared to the $>14$ day group (Figure 3C, $P_{<7 \text { day }}<0.01, P_{7 \text { to } 14 \text { day }}<0.05, P_{15}$ to 21 day $\left.<0.01\right)$.

Immunofluorescence assays were also performed for ischemic brain sections of pMCAO C57/BL6 mice. In these studies, IP-10 expressing cells were observed to localize to the ischemic penumbra, as well as to the sagittal suture, at different time points (Figure 3D,G). PCR assays demonstrated that levels of IP-10 mRNA were highest 12 hours after ischemia, especially in the ischemic hemisphere tissues (Figure $3 \mathrm{H}$ ). Furthermore, the number of NK cells present in the ischemic and contralateral hemispheres of pMCAO mouse tissues was also detected by FACS, and a similar observation was made, with a greater number of NK cells detected in ischemic tissues than contralateral tissues. Taken together, the relationship between IP-10 levels and NK levels was found to be significant (Figure 3I, $P<0.001$ ). Then we blocked IP-10 in vivo at the 12 hour time point, the number of NK cells present in the ischemic hemispheres of pMCAO mouse tissues were significantly low $(P<0.001)$ which were detected by FACS(Figure 3J, $P<0.001$ ).

In our laboratory, IP-10 has been found to be produced by local cells (glial cells and endothelial cells) in an inflammatory lesion and cells culture supernatants (data not shown). Therefore, expression of the receptor for IP-10, CXCR3, was assayed using immunohistochemistry. In mouse pMCAO brain tissue, CXCR3-positive infiltrating NK cells were detected (Figure 4A). NK cells were then 


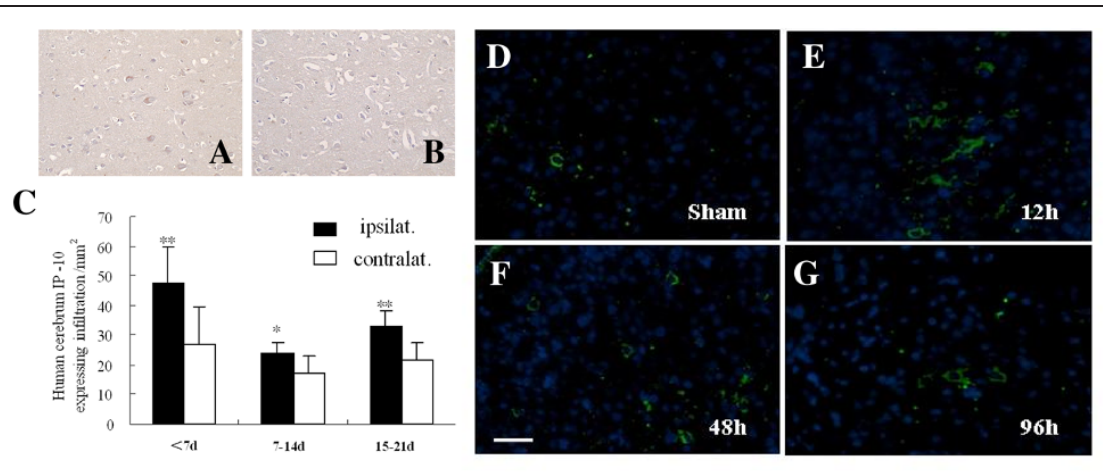

H
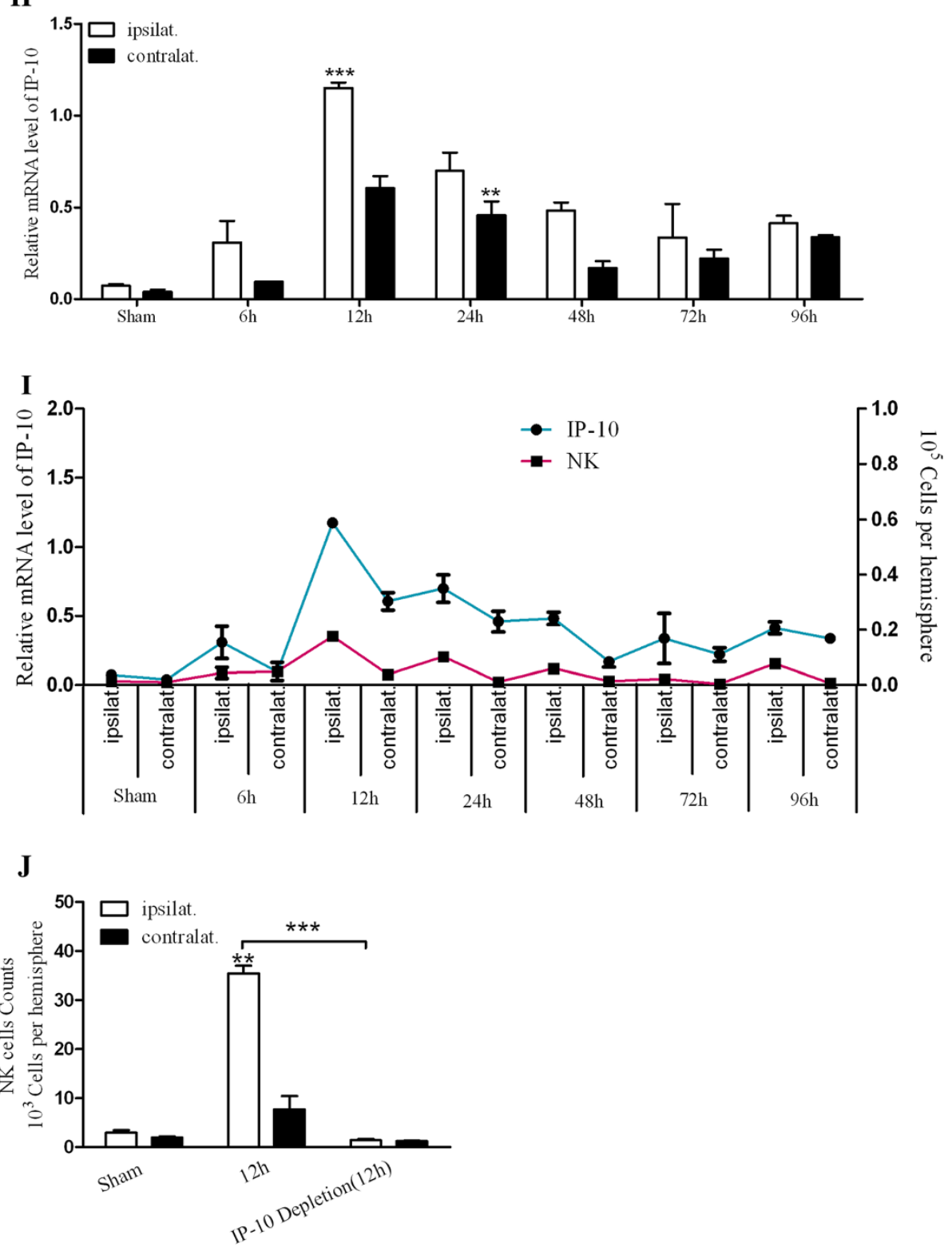

Figure $\mathbf{3}$ (See legend on next page.) 
(See figure on previous page.)

Figure 3 Chemotactic effect of IP-10 on infiltrating NK cells during cerebral ischemia. (A,B): Expression of IP-10 in ipsilesional (A) and contralesional (B) hemispheres after stroke (magnification, 400X). (C): Significant differences between ipsilesional and contralesional hemispheres were observed. (D-G): Immunofluorescence detection of IP-10-positive cells (FITC) in mouse ischemic penumbra collected at different time points (bar $=20 \mu \mathrm{m})$. (H): Levels of IP-10 mRNA detected in mouse ischemic hemispheres by PCR; (I): Interaction between IP-10 and infiltrating NK cells; (J): Infiltration of NK cells detected in mouse ischemic tissue by FACS after IP-10 depletion in vivo. ${ }^{* *} P<0.01,{ }^{* *} P<0.001$, compared with the corresponding values of the contralateral hemispheres).

treated with various concentrations of murine IP-10 (such as $10 \mathrm{ng} / \mathrm{ml}, 50 \mathrm{ng} / \mathrm{ml}, 100 \mathrm{ng} / \mathrm{ml}$, Peprotech, USA) and subjected to 6 hour OGD conditions. Detection of the mean fluorescence value for CXCR3 indicated that CXCR3 expression in $100 \mathrm{ng} / \mathrm{ml}$ group significantly increased when compared with $50 \mathrm{ng} / \mathrm{ml}$ group $(P<0.001)$, and as the concentration of IP-10 increased, the mean fluorescence value of CXCR3 got higher. Furthermore, this number was enhanced when NK cells were deprived of oxygen (Figure 4B).

\section{NK cells accelerate BBB injury via IP-10 chemotaxis}

An in vitro model of the BBB was established with brain microvascular endothelial cells plated in the upper chambers of transwells, and neural cells plated in the lower chambers. Upon reaching confluence, the medium was
A

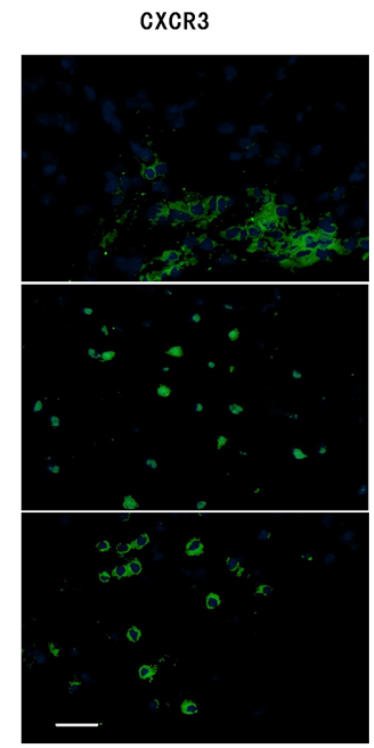

B

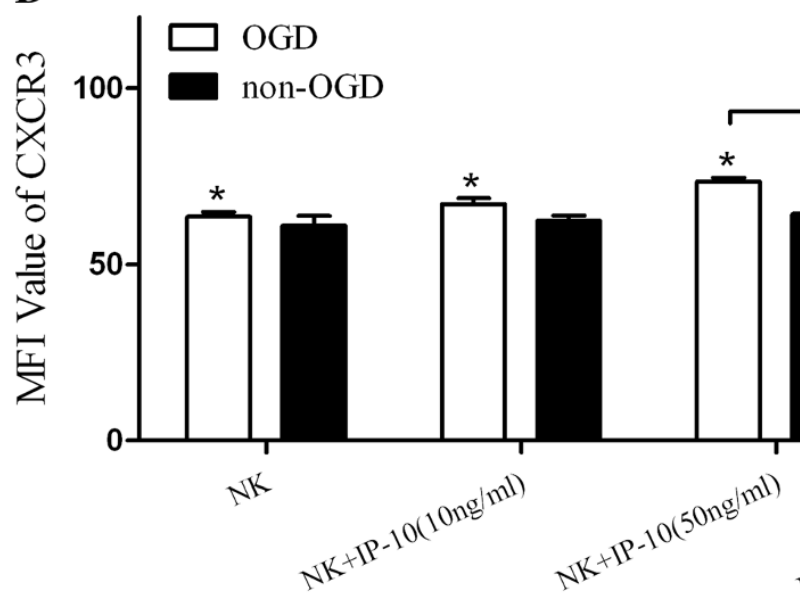

CXCR3/NKp46
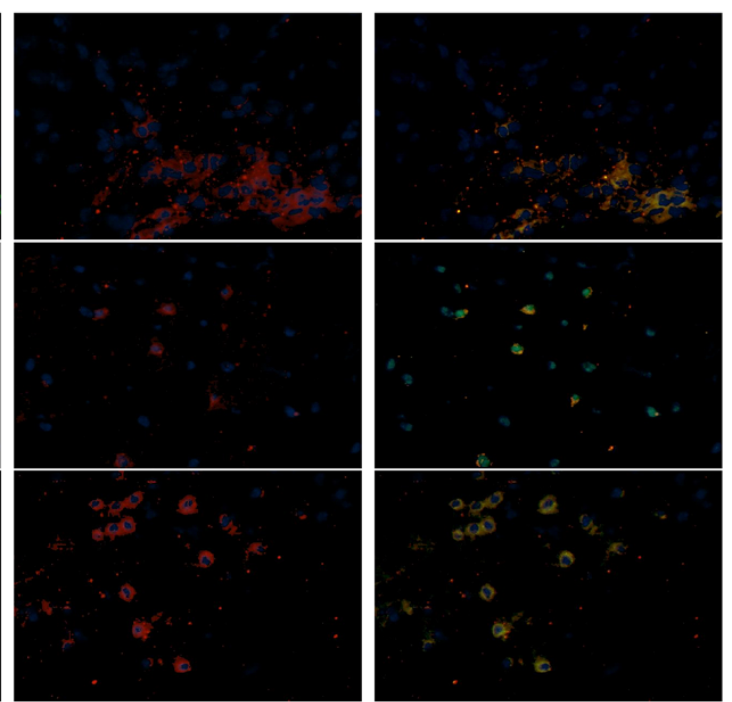

$\star \star \star$
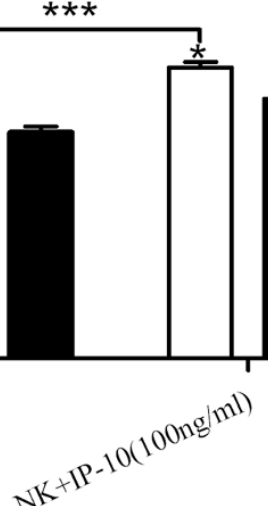

Figure 4 NK cells were absorbed by IP-10 via the linkage of CXCR3. (A) Immunofluorescence assays of NKp46 and CXCR3 in mouse ischemic cerebral tissue (bar = $20 \mu \mathrm{m})$; (B) The dose-dependent effect of IP-10 on NK cells. $\left({ }^{*} P<0.05,{ }^{* * *} P<0.001\right)$. 
changed to Ca-Mg-free HBSS (1 g/L glucose), and NK cells were added to the upper chamber. Cells were then subjected to OGD conditions. Using permeability assays, brain microvascular endothelial cells were found to be affected by the presence of NK cells after OGD (Figure 5A, $P<0.01$ ), suggesting that NK cells not only negatively affect neural cells, but may also affect the BBB during ischemia.

To investigate the effect of IP-10 on NK cells, IP-10 antibody was added to the lower chamber of the BBB model to neutralize the IP-10 secreted by neural cells (results not shown). The permeability rate of this model subsequently decreased according to the BSA diffusion assay performed (Figure 5A, $P<0.05$ ). FACS of neural cells in the lower chamber also detected a lower percentage of NK cells, thereby suggesting that the IP-10 blockade can inhibit the migration of NK cells through the $\mathrm{BBB}$ (Figure 5B, $P<0.05$ ).

\section{Discussion}

Peterfalvi et al. [16] previously demonstrated that NK cells are active during the acute phase of stroke. In addition, when NK cells were isolated from the peripheral circulation during the early phases of stroke, deficient IFN- $\gamma$ production and cytotoxicity were observed, similar to that observed in animal models [20]. Additional reports have shown that while numbers of NK cells remain unchanged following stroke, numbers of adaptive $\mathrm{T}$ lymphocyte subsets decrease [21-23]. The objective of this study was to examine the role of NK cells in brain tissue affected by ischemia. During the early phases of stroke, a peak in the infiltration of NK cells was observed, concomitant with increased expression of IFN- $\gamma$ (Figure 1A-C; Figure 2A-C). Based on these results, we hypothesized that NK cells play an important role during the onset of cerebral ischemia. In studies by Gelderblom et al. [24], NK cells were not found to significantly affect ischemia in a mouse brain 1 hour after the ischemic event and reperfusion. In the present study, numbers of infiltrating NK cells reached their highest levels in the ischemic hemisphere 12 hours after the ischemic event $(P<0.01)$ (Figure $1 \mathrm{~F})$ according to FACS analysis. Furthermore, IFN- $\gamma$-positive NK cells exhibited a similar profile (Figure 2C). The analysis of NK cells as a percentage of infiltrating lymphocytes further confirmed that NK cells have a predominant role during the onset of stroke (Figure 1G). After we depleted NK cells in vivo, NK infiltration in ischemic brain got notably lower (Figure $1 \mathrm{H}$ ) as did the level of IFN- $\gamma$ mRNA (Figure 2F), and the ischemia area diminished, which strengthens the importance of NK cells participation in the onset of cerebral ischemia.

Interestingly, when neural cells were exposed to activated NK cells, an increase in the number of dead cells and very low levels of apoptosis were observed. In contrast, a significant increase in the levels of apoptosis was detected following neutralization of IFN- $\gamma$ secreted by NK cells (Figure 2D,E). Apoptosis is currently recognized as a necessary process during the development of the nervous system [25]. Moreover, the apoptosis of neural cells is anticipated to stabilize a microenvironment more so than the presence of dead neural cells. Therefore, we hypothesize that NK cells promote the necrosis of neural cells via IFN- $\gamma$ during the early stages of stroke, and the fragments of these dead cells negatively affect the ischemic region. Accordingly, neutralization of IFN- $\gamma$ in the brain should be further investigated in clinical trials.

The detection of NK cells in ischemic brain tissue illustrates that NK cells have a role in cerebral ischemia, and the section of IFN- $\gamma$ by NK cells has the potential to affect neural cells. However, previous studies have not demonstrated how NK cells are able to access ischemic tissues. Since blood flow is blocked following an ischemic event, access via circulation is not a possibility. IP-10 is a chemoattractant secreted by several cell types in response to IFN- $\gamma$, and these cell types include monocytes,
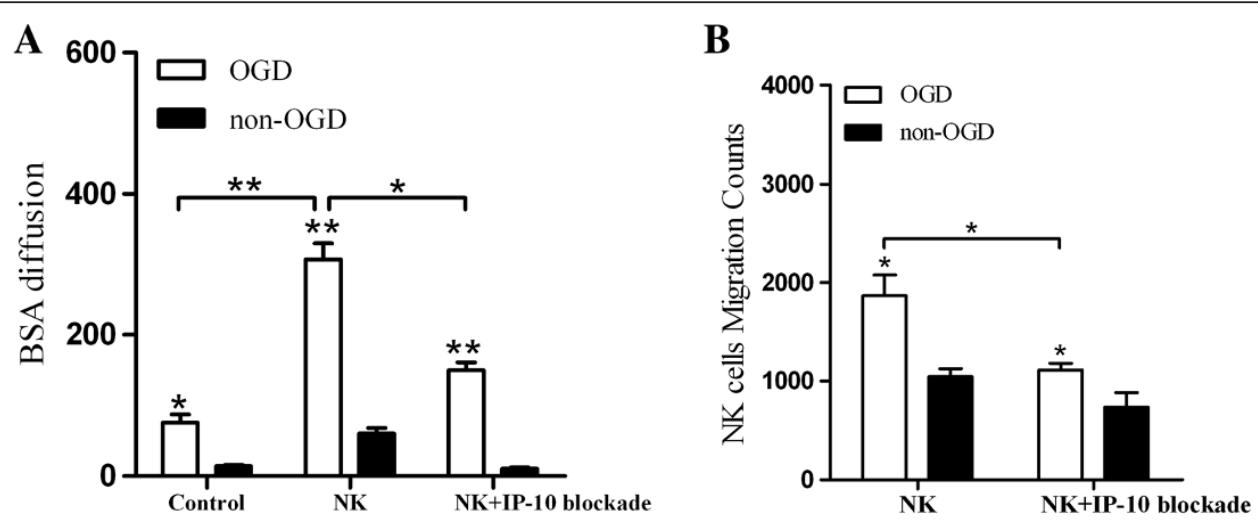

Figure 5 IP-10 promotes NK cell-induced BBB injury. (A): IP-10 blockade attenuated a BBB permeability injury induced by NK cells under OGD treatment. (B): Migration of NK cells with or without IP-10 blockade with OGD treatment. $\left.{ }^{*} P<0.05,{ }^{* *} P<0.01\right)$. BBB, blood brain barrier; OGD, oxygen glucose deprivation. 
endothelial cells, and fibroblasts [26]. Furthermore, IP-10 has been identified as a chemoattractant for monocytes and macrophages, T cells, NK cells, and dendritic cells [27]. IP-10 has been proven to increase in focal stroke [28-30]. In our study, we focused on probing the relationship between IP-10 and NK cells accumulation after stroke. IP-10 was detected in human ischemic brain tissue (Figure $3 \mathrm{~A}, \mathrm{~B}$ ), and was present at high levels during the early stages of stroke (Figure $3 \mathrm{C}$ ). Similarly, IP-10 was detected in ischemic cerebral tissue of the mouse model examined (Figure 3D,G), with the highest levels detected 12 hours after ischemia using RT-PCR. These results are consistent with the rat model of ischemia characterized by Wang et al. [31] (Figure 3H), and the decrease of NK cells infiltration in ischemic brain after IP-10 depletion in vivo prompt us to compare relationship between IP-10 and NK cells when stroke occurs, suggesting that IP-10 expression induces NK cell infiltration following cerebral ischemia (Figure 3I,J).

CXCR3 regulates leukocyte trafficking, and binding of chemokines such as IP-10 can induce various cellular responses [32]. Most notably, these changes can include integrin activation, cytoskeletal changes, and chemotactic migration. However, these responses are restored following the dephosphorylation of intracellular receptors and their subsequent recycling to the cell surface. In the present study, detection of mean fluorescence intensity was used to evaluate the action of IP-10 on NK cells. As a result, IP-10 was found to activate CXCR3 expression by NK cells (Figure 4A), and this effect was dose-dependent (Figure 4B). However, IFN- $\gamma$-mediated cytoxicity associated with IP-10-induced activation of NK cells was not observed (data not shown). Therefore, it appears that IP-10 is only a chemotactic cytokine for NK cells following cerebral ischemia.

NK cells respond to several chemotactic factors, including MIP-1 and Mig [33]. However, IP-10 is the primary chemotactic factor for NK cells in the brain. In the present study, IP-10 was found to be primarily secreted by neural cells rather than brain microvascular endothelial cells under OGD conditions (data not shown). In the brain, the stability of the microenvironment is maintained by $\mathrm{BBB}$ permeability [34]. Correspondingly, damage to the BBB can promote secondary inflammation injury following stroke [35]. By co-culturing neural cells and cerebral microvascular endothelial cells under OGD conditions as an analogue of ischemia and the BBB, an increase in permeability was observed following the addition of NK cells (Figure 5A). Furthermore, this increase in permeability, as well as the migration of NK cells, was reduced following neutralization of IP-10 (Figure 5B). These results strongly suggest that NK cells negatively affect the $\mathrm{BBB}$ during stroke, and IP-10 enhances the infiltration of NK cells through the $\mathrm{BBB}$ following cerebral ischemia.

\section{Conclusions}

In summary, NK cells were found to participate in the early stages of stroke and to induce the necrosis of neural cells via IFN- $\gamma$. Based on these results, the capacity to neutralize IFN- $\gamma$ represents an opportunity to stabilize the microenvironment associated with cerebral ischemia. In addition, NK cells were found to damage the BBB in response to IP-10 as a chemoattractant. These results suggest that the damage mediated by NK cells following stroke may be attenuated by neutralizing IP-10 and other factors that are attractive to NK cells, thereby preventing the release of cytotoxic cytokines. It is anticipated that further studies of these mechanisms may improve the indications of clinical therapy for brain ischemia. Furthermore, future studies will need to focus on NK cells present in ischemic tissue and their interactions with other immune cells during different phases of stroke, particularly interactions that lead to damage of the BBB.

\section{Abbreviations}

TTC: 2,3,5-triphenyltetrazolium chloride; pMCAO: permanent middle cerebral artery occlusion; BBB: blood brain barrier; OGD: oxygen glucose deprivation; PBS: Phosphate Buffered Saline; HBSS: Hank's Balanced Salt Solution; ANOVA: analysis of variance; DMEM: Dulbecco's-modified Eagle's medium; FBS: fetal bovine serum; OGD: oxygen-glucose deprivation; PI: propidium iodide.

\section{Competing interests}

The authors declare that they have no competing interests.

\section{Authors' contributions}

$Y Z, Z G, G W$ and $H L$ designed research, performed research, analyzed data, and wrote the manuscript. DW, TZ, BS, LM, JW, YL, QK, XL, YZ, HZ and JH performed research, analyzed data, and wrote the manuscript. All authors read and approved the final manuscript.

\section{Acknowledgments}

This research was supported by National Nature Science Foundation of China (81070943); National Nature Science Foundation of China (31371079); Natural Science Foundation of Heilongjiang Province (QC2013C082); Heilongjiang Provincial Department of Education Science and Technology Research Project (12531393); Heilongjiang Provincial Education Department 2011 Annual New Century Excellent Talents (1251-NCET-010); Open Topic of Key Laboratory of Myocardial Ischemia, Harbin Medical University Ministry of Education

Heilongjiang Province of China (KF201206); Open Topic of Key Laboratory of Neurobiology, General Colleges and Universities in Heilongjiang Province of China (2012HUKLNT-09); and Open Topic of Key Laboratory of Neurobiology, General Colleges and Universities in Heilongjiang Province of China (2012HLKLNT-01).

\section{Author details}

${ }^{1}$ Department of Neurobiology, Harbin Medical University Provincial Key Lab of Neurobiology, Harbin Medical University, Xuefu Road, 150081, Heilongjiang, People's Republic of China. ${ }^{2}$ Neurology department, The Affiliated Hospital of Hangzhou Normal University, Wenzhou Road, 310015 Zhejiang, People's

Republic of China. ${ }^{3}$ The Key Laboratory of Myocardial Ischemia, Harbin Medical University, Ministry of Education, Xuefu Road, 150001, Heilongjiang, Province People's Republic of China. ${ }^{4}$ Key Lab of Magnetic Resonance Imaging Device and Technique, Huayuanbei Road, 100082 Beijing, People's Republic of China. ${ }^{5}$ Department of Otorhinolaryngology, Mudanjiang 2nd People's Hospital, Guanghua Road, 157005, Heilongjiang, People's Republic of China.

Received: 6 November 2013 Accepted: 5 April 2014

Published: 17 April 2014 


\section{References}

1. Farina $C$, Aloisi F, Meinl E: Astrocytes are active players in cerebral innate immunity. Trends Immunol 2007, 28:138-145.

2. Wilson DR, Warise L: Cytokines and their role in depression. Perspect Psychiatr Care 2008, 44:285-289.

3. London A, Itskovich E, Benhar I, Kalchenko V, Mack M, Jung S, Schwartz M: Neuroprotection and progenitor cell renewal in the injured adult murine retina requires healing monocyte-derived macrophages. J Exp Med 2011, 208:23-39.

4. Madrigal $J \mathrm{~L}$, Leza JC, Polak P, Kalinin S, Feinstein DL: Astrocyte-derived MCP-1 mediates neuroprotective effects of noradrenaline. $J$ Neurosci 2009, 29:263-267.

5. Dirnagl U, ladecola C, Moskowitz MA: Pathobiology of ischaemic stroke: an integrated view. Trends Neurosci 1999, 22:391-397.

6. Huang J, Upadhyay UM, Tamargo RJ: Inflammation in stroke and focal cerebral ischemia. Surg Neurol 2006, 66:232-245.

7. Garcia JH, Liu KF, Yoshida Y, Lian J, Chen S, Del Zoppo GJ: Influx of leukocytes and platelets in an evolving brain infarct (Wistar rat). Am J Pathol 1994, 144:188-199.

8. Lipton P: Ischemic cell death in brain neurons. Physiol Rev 1999, 79:1431-1568.

9. Vivier E, Raulet DH, Moretta A, Caligiuri MA, Zitvogel L, Lanier LL, Yokoyama WM, Ugolini S: Innate or adaptive immunity? The example of natural killer cells. Science 2011, 331:44-49.

10. Robertson MJ, Ritz J: Biology and clinical relevance of human natural killer cells. Blood 1990, 76:2421-2438.

11. Cooper MA, Fehniger TA, Caligiuri MA: The biology of human natural killer-cell subsets. Trends Immunol 2001, 22:633-640.

12. Strowig T, Brilot F, Munz C: Noncytotoxic functions of NK cells: direct pathogen restriction and assistance to adaptive immunity. J Immunol 2008, 180:7785-7791.

13. Bielekova B, Catalfamo M, Reichert-Scrivner S, Packer A, Cerna M, Waldmann TA, McFarland H, Henkart PA, Martin R: Regulatory CD56 bright natural killer cells mediate immunomodulatory effects of IL-2Ra-targeted therapy (daclizumab) in multiple sclerosis. Proc Natl Acad Sci 2006, 103:5941-5946.

14. Beum PV, Lindorfer MA, Taylor RP: Within peripheral blood mononuclear cells, antibody-dependent cellular cytotoxicity of rituximab-opsonized Daudi cells is promoted by NK cells and inhibited by monocytes due to shaving. J Immunol 2008, 181:2916-2924.

15. Yabuhara A, Yang F-C, Nakazawa T, Iwasaki Y, Mori T, Koike K, Kawai H, Komiyama A: A killing defect of natural killer cells as an underlying immunologic abnormality in childhood systemic lupus erythematosus. J Rheumatol 1996, 23:171.

16. Peterfalvi A, Molnar T, Banati M, Pusch G, Miko E, Bogar L, Pal J, Szereday L, Illes Z: Impaired function of innate T lymphocytes and NK cells in the acute phase of ischemic stroke. Cerebrovasc Dis 2009, 28:490-498.

17. Minematsu K, Li L, Sotak CH, Davis MA, Fisher M: Reversible focal ischemic injury demonstrated by diffusion-weighted magnetic resonance imaging in rats. Stroke 1992, 23:1304-1310.

18. Bederson JB, Pitts LH, Germano SM, Nishimura MC, Davis RL, Bartkowski HM: Evaluation of 2,3,5-triphenyltetrazolium chloride as a stain for detection and quantification of experimental cerebral infarction in rats. Stroke 1986, 17:1304-1308

19. Kebir H, Kreymborg K, Ifergan I, Dodelet-Devillers A, Cayrol R, Bernard M, Giuliani F, Arbour N, Becher B, Prat A: Human TH17 lymphocytes promote blood-brain barrier disruption and central nervous system inflammation. Nat Med 2007, 13:1173-1175.

20. Prass K, Meisel C, Hoflich C, Braun J, Halle E, Wolf T, Ruscher K, Victorov IV, Priller J, Dirnagl U, Volk HD, Meisel A: Stroke-induced immunodeficiency promotes spontaneous bacterial infections and is mediated by sympathetic activation reversal by poststroke T helper cell type 1-like immunostimulation. J Exp Med 2003, 198:725-736.

21. Vogelgesang A, Grunwald U, Langner S, Jack R, Broker BM, Kessler C, Dressel $A$ : Analysis of lymphocyte subsets in patients with stroke and their influence on infection after stroke. Stroke 2008, 39:237-241.

22. Klehmet J, Harms H, Richter M, Prass K, Volk HD, Dirnagl U, Meisel A, Meisel C: Stroke-induced immunodepression and post-stroke infections: lessons from the preventive antibacterial therapy in stroke trial. Neuroscience 2009, 158:1184-1193.

23. Urra X, Cervera A, Villamor N, Planas AM, Chamorro A: Harms and benefits of lymphocyte subpopulations in patients with acute stroke. Neuroscience 2009, 158:1174-1183.
24. Gelderblom M, Leypoldt F, Steinbach K, Behrens D, Choe CU, Siler DA, Arumugam TV, Orthey E, Gerloff C, Tolosa E, Magnus T: Temporal and spatial dynamics of cerebral immune cell accumulation in stroke. Stroke 2009, 40:1849-1857.

25. Yuan J, Yankner BA: Apoptosis in the nervous system. Nature 2000, 407:802-809.

26. Luster AD, Unkeless JC, Ravetch JV: Gamma-interferon transcriptionally regulates an early-response gene containing homology to platelet proteins. Nature 1985, 315:672-676.

27. Dufour JH, Dziejman M, Liu MT, Leung JH, Lane TE, Luster AD: IFN-gammainducible protein 10 (IP-10; CXCL10)-deficient mice reveal a role for IP-10 in effector T cell generation and trafficking. J Immunol 2002, 168:3195-3204.

28. Li HL, Kostulas N, Huang YM, Xiao BG, van der Meide P, Kostulas V, Giedraitas $V$, Link H: IL-17 and IFN-gamma mRNA expression is increased in the brain and systemically after permanent middle cerebral artery occlusion in the rat. J Neuroimmunol 2001, 116:5-14.

29. Chaitanya GV, Eeka P, Munker R, Alexander JS, Babu PP: Role of cytotoxic protease granzyme-b in neuronal degeneration during human stroke. Brain Pathol 2011, 21:16-30.

30. Chaitanya GV, Schwaninger M, Alexander JS, Babu PP: Granzyme-b is involved in mediating post-ischemic neuronal death during focal cerebral ischemia in rat model. Neuroscience 2010, 165:1203-1216.

31. Wang X, Ellison JA, Siren AL, Lysko PG, Yue TL, Barone FC, Shatzman A, Feuerstein GZ: Prolonged expression of interferon-inducible protein-10 in ischemic cortex after permanent occlusion of the middle cerebral artery in rat. J Neurochem 1998, 71:1194-1204.

32. Wang X, Li X, Schmidt DB, Foley JJ, Barone FC, Ames RS, Sarau HM: Identification and molecular characterization of rat CXCR3: receptor expression and interferon-inducible protein-10 binding are increased in focal stroke. Mol Pharmacol 2000, 57:1190-1198.

33. Robertson MJ: Role of chemokines in the biology of natural killer cells. J Leukoc Biol 2002, 71:173-183.

34. Abbott NJ: Blood-brain barrier structure and function and the challenges for CNS drug delivery. J Inherit Metab Dis 2013, 36:437-449.

35. Yang G-Y, Betz AL: Reperfusion-induced injury to the blood-brain barrier after middle cerebral artery occlusion in rats. Stroke 1994, 25:1658-1664.

doi:10.1186/1742-2094-11-79

Cite this article as: Zhang et al:: Accumulation of natural killer cells in ischemic brain tissues and the chemotactic effect of IP-10. Journal of Neuroinflammation 2014 11:79.

\section{Submit your next manuscript to BioMed Central and take full advantage of:}

- Convenient online submission

- Thorough peer review

- No space constraints or color figure charges

- Immediate publication on acceptance

- Inclusion in PubMed, CAS, Scopus and Google Scholar

- Research which is freely available for redistribution

Submit your manuscript at www.biomedcentral.com/submit
C) Biomed Central 